\title{
Labor Pattern in South Indian Population
}

\author{
Chitra Bhat ${ }^{1}$, Seetha Panicker ${ }^{2}$
}

\begin{abstract}
Aim: Labor dystocia is one of the commonest indications of lower segment cesarean section (LSCS). Recent studies have shown a dramatic change in the rate of cervical dilatation and labor progress. There are no large studies to assess labor in this ethnic group and hence this study has been done to find out labor behavior in women with spontaneous labor in South India.

Materials and methods: A retrospective study was done in our teaching hospital. Labor was managed as per protocol and a partogram was maintained. Data were extracted from case-records and analyzed.

Results: Labor progress was much slower than what was thought of. There was wide variability in the duration of labor.

Conclusion: Many women took time up to the 95th percentile and some even crossed three times the median time to deliver.

Clinical significance: Though many women will deliver in the expected time, it is important to know the maximum time or the 95th percentile for each dilatation so that sufficient time can be allowed before labeling a case as prolonged labor.

Keywords: Labor curve, Labor duration, Rate of cervical dilatation, Slowest rate of dilatation.

Journal of South Asian Federation of Obstetrics and Gynaecology (2020): 10.5005/jp-journals-10006-1839
\end{abstract}

\section{INTRODUCTION}

Cervical dilatation is one of the key determinants of the progress of labor and may be dependent on many factors. There have been many attempts to define the norms for the progress of labor that have yielded variable results.

This is not an easy task as there are many challenges to accurately measure the duration of labor. The labor norms are also dependent on a woman's ethnicity, age, parity, BMI, use of epidural analgesia, and may vary among population groups. Despite all these difficulties, it is important to arrive at a proper understanding of labor norms and identify the slowest acceptable rates of cervical dilatation.

The rates of cervical dilatation in the active phase of labor largely stem from the data published by Friedman in 1950. ${ }^{1}$ The Friedman's labor curve and the active management of labor pioneered by O'Driscoll et al. ${ }^{2}$ where the lowest acceptable rate of dilatation is $1 \mathrm{~cm} / \mathrm{hr}$ has influenced obstetric practice worldwide. WHO partogram ${ }^{3}$ is practiced all over the world and the expected rate of dilatation is $1 \mathrm{~cm} / \mathrm{hr}$ in the active phase. Any shift to the right and if the curve crosses the action line, labor is labeled as prolonged labor or non-progression of labor, and lower segment cesarean section (LSCS) is performed.

Dystocia characterized by a delay in progression of cervical dilatation is one of the most common indications for primary cesarean sections, ACOG Bulletin states it is the leading cause contributing to around $50 \%$ of all primary LSCS being performed. ${ }^{4}$

Zhang et al. reassessed Friedman's curves. ${ }^{5}$ They studied the electronic records of 62,416 parturients and found the rates of cervical dilatation to be much slower. Data from this study formed the basis of the new guidelines for the active phase of labor. Labor may take even 6 hours to progress from $4-5 \mathrm{~cm}$ and more than 3 hours to progress from $5-6 \mathrm{~cm}$. Only after $6 \mathrm{~cm}$ was the progress faster.

Due to ethnic differences, it is important to have insight regarding labor in our population that is critical in diagnosing labor dystocia and making a decision regarding the mode of delivery

\footnotetext{
1,2Department of Obstetrics and Gynaecology, PSG Institute of Medical Sciences and Research, Coimbatore, Tamil Nadu, India

Corresponding Author: Chitra Bhat, Department of Obstetrics and Gynaecology, PSG Institute of Medical Sciences and Research, Coimbatore, Tamil Nadu, India, Phone: +91 9443353191, e-mail: chitrabhat63@gmail.com

How to cite this article: Bhat C, Panicker S. Labor Pattern in South Indian Population. J South Asian Feder Obst Gynae 2020;12(6):372-375.

Source of support: Nil

Conflict of interest: None
}

\section{Aims and Objectives}

- To determine the duration of labor and the rates of cervical dilatation in low-risk primigravida and multigravida women with spontaneous onset of labor.

- To determine the slowest acceptable rates of cervical dilatation.

- To create a labor curve for this population.

\section{Materials and Methods}

This was a prospective observational study done in a teaching hospital in South India between 2017 and 2018.

The study population included both primigravida and multigravida who were admitted with spontaneous labor.

The inclusion criteria were term gestation, singleton pregnancy, and vertex presentation with spontaneous onset of labor who delivered either normally or by assisted vaginal delivery. The exclusion criteria were induced labor, pre-term labor, malpresentations, and patients who were delivered by cesarean section.

The institutional protocols for monitoring of labor were followed. Maternal and fetal monitoring was done in the usual way. The progress of labor was assessed by monitoring uterine contractions per 10 minutes for every half an hour, cervical

(0) Jaypee Brothers Medical Publishers. 2020 Open Access This article is distributed under the terms of the Creative Commons Attribution 4.0 International License (https://creativecommons.org/licenses/by-nc/4.0/), which permits unrestricted use, distribution, and non-commercial reproduction in any medium, provided you give appropriate credit to the original author(s) and the source, provide a link to the Creative Commons license, and indicate if changes were made. The Creative Commons Public Domain Dedication waiver (http://creativecommons.org/publicdomain/zero/1.0/) applies to the data made available in this article, unless otherwise stated. 
dilatation, and descent of the head was assessed by vaginal examination every 3-4 hours in the first stage of labor.

The rate of cervical dilatation was plotted on a partogram with time in hours on the $x$-axis and cervical dilatation on the $y$-axis.

Ethical clearance was obtained from our institutional human ethical committee.

\section{Statistical Analysis}

The data was entered on an Excel spreadsheet and analysis was done using SPSS STATA13.1 software.

The data were analyzed concerning maternal demographic characteristics. The cumulative labor duration from cervical dilatation on admission (i.e., at 1, 2, 3, 4, 6, 7) to reach $10 \mathrm{~cm}$ was assessed. Data were extracted according to two parity groups nulliparous and parous women.

With each starting dilatation, the mean rate of dilatation was calculated for primigravida and multigravida. Taking the value of $2 \mathrm{SD}$, the slowest rate of dilatation was also calculated.

A non-regression model was used to plot the labor curve with cervical dilatation against time in hours.

\section{Results}

The total number of deliveries during the study period was 4150 .

A total of 2558 women were delivered by cesarean section. The number of women with spontaneous onset of labor who delivered vaginally was 1196 . A total of 152 patients who had incomplete data and 21 patients who were admitted at more than $8 \mathrm{~cm}$ were excluded. So, only 1023 deliveries were analyzed.

Primigravida were 556 in number and multigravida were 467 in number. The age, cervical findings on admission, and mode of delivery are shown in Table 1.

The cumulative time taken for each dilatation starting from 1 to reach $10 \mathrm{~cm}$ was analyzed in both primigravida and multigravida.

In our study, the median duration of labor was chosen, as the mean duration may be skewed by a few laborers of very short or long duration. If the labor duration is positively skewed, the mean duration will be longer than the median duration as shown in this study thus at $2 \mathrm{~cm}$ the mean duration was 7.7 hours and the median duration was 7 hours.
Table 2 shows the cumulative mean time taken for each dilatation to reach $10 \mathrm{~cm}$ in primigravida and multigravida, respectively. It shows that multigravidae took a shorter time to progress to $10 \mathrm{~cm}$ when compared to primigravida. Primigravida took a median time of 9 hours to reach $10 \mathrm{~cm}$ from $1 \mathrm{~cm}$, whereas it was 8 hours in multiparous women. At $2 \mathrm{~cm}$ it was 7 and 6 hours. At 3, 4, 5, 6-10 cm it was 5, 3, 3, and 2 hours in primigravida and 4, 3,2 , and 2 hours in multigravida.

Table 2 also shows the 5 th and the 95th percentile, the number of women who crossed the 95th percentile, and the maximum time allowed for labor to continue that resulted in normal vaginal delivery.

At $2 \mathrm{~cm}$ though the meantime was 7.7 hour in primigravida, 16 women crossed the 95 th percentile and 10 patients had a vaginal delivery after almost three times this mean-ranging from 18-26 hours. In six multiparous women at the same dilatation, labor was prolonged to 16-27 hours and 15 women crossed the 95th percentile.

At $3 \mathrm{~cm}$ dilatation, although the mean duration of labor was 5.5 hours, four primigravida women had a vaginal delivery after $19,17,16$, and 13 hours, respectively. Correspondingly three multigravidas had taken 21,15 , and 13 hours to deliver from $3 \mathrm{~cm}$ dilatation for a mean of 4.7 hours.

At $4 \mathrm{~cm}$ against a mean of 3.8 hours, five primigravidas had taken between 8 and 11 hours to deliver, and in multigravida for a mean of 2.9 hours four had taken between 6 and 9 hours. At $7 \mathrm{~cm}$ a multiparous woman took 6 hours to reach full dilatation.

With each starting dilatation, the mean rate of dilatation was calculated for primis and multis. Taking the value of 2SD, the slowest rate of dilatation was also calculated as shown in Table 3.

The labor curve obtained was hyperbolic and not a sigmoid curve. There was no definite point of acceleration seen (Figs 1 and 2).

Table 1: Demographics

\begin{tabular}{lll}
\hline Details & Primigravida & Multigravida \\
\hline Number of cases & 556 & 467 \\
Age & 24.2 years & 27.4 years \\
$\%$ of normal delivery & $74.3 \%$ & $87.9 \%$ \\
Instrumental & $25.7 \%$ & $12.1 \%$ \\
Mean dilatation on admission & $2.85 \mathrm{~cm}$ & $3.2 \mathrm{~cm}$ \\
\hline
\end{tabular}

Table 2: Cumulative duration of labor (dilatation on admission to $10 \mathrm{~cm}$ )

\begin{tabular}{|c|c|c|c|c|c|c|c|c|c|}
\hline Dilatation on admission $(\mathrm{cm})$ & Obs score & Numbers & Mean & Median & $S D$ & P5 & P95 & No $>95$ & Max time taken \\
\hline \multirow[t]{2}{*}{$1-2$} & Primi & 121 & 9.36 & 9 & 5.05 & 2.5 & 19 & 17 & 30 \\
\hline & Multi & 84 & 9.39 & 8 & 5 & 2.5 & 19 & 16 & 27 \\
\hline \multirow[t]{2}{*}{$2-3$} & Primi & 201 & 7.7 & 7 & 4.5 & 2 & 16 & 10 & 26 \\
\hline & Multi & 158 & 6.8 & 6 & 4.2 & 2 & 15 & 6 & 27 \\
\hline \multirow[t]{2}{*}{$3-4$} & Primi & 112 & 5.5 & 5 & 3.2 & 2 & 10 & 5 & 19 \\
\hline & Multi & 122 & 4.7 & 4 & 3.1 & 1 & 10 & 5 & 21 \\
\hline \multirow[t]{2}{*}{$4-5$} & Primi & 69 & 3.8 & 3 & 2.2 & 1 & 8 & 3 & 11 \\
\hline & Multi & 74 & 2.9 & 3 & 1.6 & 1 & 6 & 2 & 9 \\
\hline \multirow[t]{2}{*}{$5-6$} & Primi & 29 & 3 & 3 & 1.8 & 1 & 6 & 1 & 10 \\
\hline & Multi & 30 & 2.2 & 2.2 & 1.7 & 1 & 6 & 1 & 8 \\
\hline \multirow[t]{2}{*}{$6-7$} & Primi & 12 & 1.8 & 2 & 0.68 & 1 & 3 & - & - \\
\hline & Multi & 6 & 1.8 & 2 & 0.68 & 1 & 3 & - & - \\
\hline \multirow[t]{2}{*}{$7-8$} & Primi & 12 & 1.4 & 1 & 0.64 & - & 3 & - & - \\
\hline & Multi & 21 & 1.4 & 1 & 1.13 & - & 3 & 1 & 6 \\
\hline
\end{tabular}




\begin{tabular}{llll}
\multicolumn{2}{l}{ Table 3: Rate of cervical dilatation } \\
\hline \multirow{2}{*}{ Dilatation $(\mathrm{cm})$} & Obs score & $\begin{array}{l}\text { Mean rate of } \\
\text { dilatation }(\mathrm{cm})\end{array}$ & $\begin{array}{l}\text { Slowest rate of } \\
\text { dilatation }(\mathrm{cm})\end{array}$ \\
\hline $2-3$ & Primi & 1.03 & 0.65 \\
& Multi & 1.17 & 0.72 \\
$3-4$ & Primi & 1.27 & 0.8 \\
& Multi & 1.48 & 0.89 \\
$4-5$ & Primi & 1.57 & 1 \\
& Multi & 2.06 & 1.33 \\
$5-6$ & Primi & 1.66 & 1.04 \\
& Multi & 2.27 & 1.28 \\
$6-7$ & Primi & 2.2 & 1.61 \\
& Multi & 2.2 & 1.61
\end{tabular}

\section{Discussion}

A total of 1023 patients were analyzed, out of which 556 were primigravida and 467 were multigravida. We found the following in our study:

- In the active phase, the average time taken was more than that described by Friedmann. ${ }^{6,7}$ In his study, he has stated that from $2.5 \mathrm{~cm}$ to reach $10 \mathrm{~cm}$ the average time is $4.6-4.9$ hours, and once they reach $4 \mathrm{~cm}$ half of them deliver in 2.6 hours. Our corresponding figures were 7.7 hours to reach $10 \mathrm{~cm}$ from $2.5 \mathrm{~cm}$ and the mean duration at $4 \mathrm{~cm}$ was 3.8 hours against 2.6 hours. Peisner and Rosen $^{8}$ found that $75 \%$ of women at $3 \mathrm{~cm}, 50 \%$ at $4 \mathrm{~cm}$, and $25 \%$ at $5 \mathrm{~cm}$ do not dilate at the indicated rates associated with active labor.

However, recent studies have shown a longer first stage of labor with slower acceptable rates of dilatation. Zhang et al. derived the average labor curves from 27,170 nulliparous women in the US population with spontaneous onset of labor. The curve had a slower progression of $1.1 \mathrm{~cm} / \mathrm{hr}$ with no definite inflection point. The slowest acceptable rate was $0.6 \mathrm{~cm} / \mathrm{hr}$.

Albers et al., ${ }^{9}$ Albers, ${ }^{10}$ and Jones and Larson ${ }^{11}$ found that labor progression takes a longer time than the thought of earlier. These investigators have reported that in the active phase of labor from $4 \mathrm{~cm}$ to reach $10 \mathrm{~cm}$ the time ranges from 6.2-7.7 hours on average. In Albers's study, the time taken was 7.7 hours since there was no use of oxytocin and labor was spontaneous.

- The second derivative was that there was a wide difference in time taken between individuals. When women were admitted at $2 \mathrm{~cm}$, the median time taken to reach $10 \mathrm{~cm}$ was 7 hours in primigravida and 6 hours in multigravida. However, 2SD at this dilatation was 4.5 hours extending labor to 11.5 hours. Many women crossed the 95th percentile and also, ten primigravidas delivered normally after a duration that was almost three times the median ranging from 18-26 hours indicating wide variability in the duration of labor are still associated with normal outcomes. The same was seen at all dilatations. Our findings were similar to that quoted by Oladapo et al. ${ }^{12}$ as part of WHO BOLD PROJECT (better outcome in labor difficulty) where he says median cumulative duration of labor indicates that nulliparous women admitted at 4, 5, and $6 \mathrm{~cm}$ reached $10 \mathrm{~cm}$ within an expected time, but their corresponding 95th percentile shows that labor could last up to 14, 11, and 10 hours.

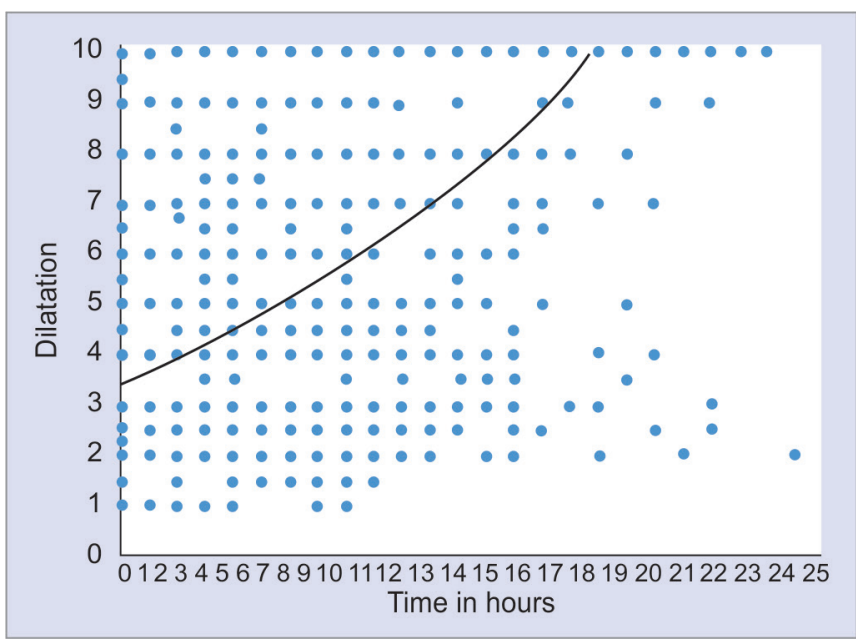

Fig. 1: Labor curve-primigravida

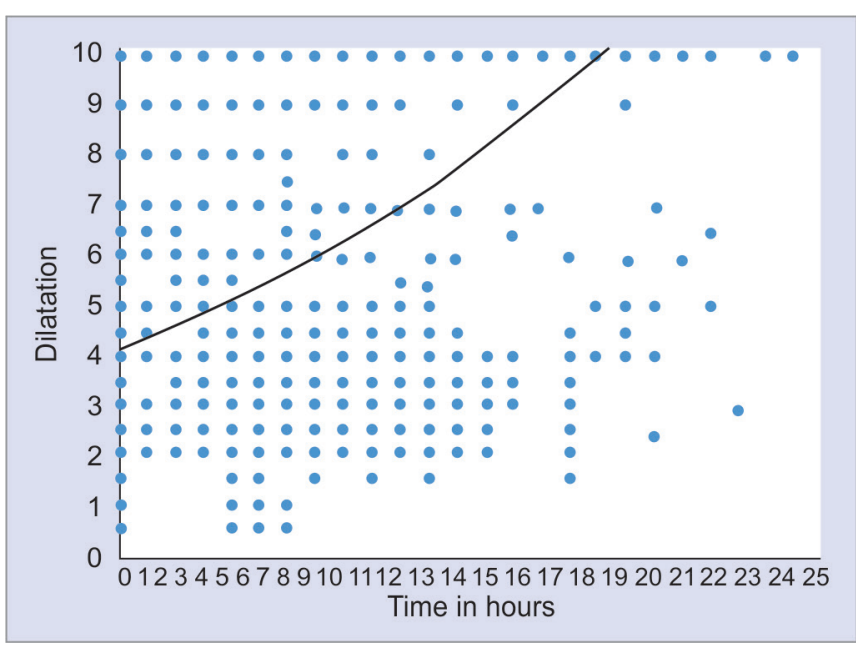

Fig. 2: Labor curve-multigravida

- It was found that the rates of cervical dilatation accelerate as labor progresses and for each dilatation, an acceptable slow rate of progress exists. This is comparable to the study by Zhang et al. who found that the slopes of cervical dilatation progressively steepen with each passing centimeter. In his study, he also found that the median rates of dilatation between $3-4,4-5,5-6,6-7$, $7-8,8-9$, and $9-10 \mathrm{~cm}$ were $0.4,0.6,1.2,1.7,2.2,2.4$, and $2.4 \mathrm{~cm}$, respectively. At the 5 th percentile that is used to define the slowest normal dilatation rates the rates were $0.1,0.2,0.3,0.5$, $0.7,0.8$, and $0.7 \mathrm{~cm} /$ hour, respectively.

- Finally, the labor curve that was obtained showed a smooth hyperbolic curve, there was no point of acceleration. A similar labor curve pattern is seen in other studies. Suzuki et al. ${ }^{13}$ studied the labor patterns in 2369 low-risk nulliparous Japanese women with spontaneous onset of labor and derived the labor curve for that population. It showed that the curve had a smooth shape with an active phase duration of 5.1 hours with slower progression than that of Friedman's curve. The deceleration phase was not observed and the rate of dilatation started to double from 5-6 cm onwards. A study done in South India by Pitchaimuthu and Bhaskaran ${ }^{14}$ showed the same pattern 
of slower dilatation, a smooth labor curve, and no point of inflection.

The limitations of this study include interpersonal variations in the assessment of cervical dilatation. The effect of ARM and oxytocin were not analyzed since many patients were subjected to either both or one of them.

\section{CONCLUSION}

In low-risk women, both primigravida and multigravida with spontaneous onset of labor, there is wide variability in the duration of labor with normal maternal and fetal outcomes. Our study showed that in low-risk women admitted in early spontaneous labor, half of them took more than the meantime and a significant number of women took even three times the mean. The rate gradually increased with dilatation and in nulliparous women at $6 \mathrm{~cm}$ of dilatation the rate was much higher. It is worthwhile to note that many women despite crossing the 95th percentile delivered normally, hence, revision of the expectations of the rate of cervical dilatation would reduce both unnecessary interventions in labor and cesarean sections for slow or abnormal progress of labor. Labor should be more patient-centered and the same pattern will not fit all.

\section{Acknowledgments}

I sincerely thank both of the statisticians Dr. Varun and Dr. Karthikeyan who took great efforts in analyzing the data and deriving the labor curve. I thank all the staff involved to retrieve the case sheets and the junior residents who helped me with data entry.

\section{References}

1. Friedman EA. Primigravid labour: a graphicostatical analysis. Obstet Gynecol 1955;6(6):567-589. DOI: 10.1097/00006250-19551200000001. Pubmed:13272981.
2. O'Driscoll K, Stronge JM, Minogue M. Active management of labour Br Med J 1973;3(5872):135-137. DOI: 10.1136/bmj.3.5872.135. Pubmed: 4720762.

3. World Health Organizaton. Division of family health. Maternal health and safe motherhood programme. The partograph; the application of the WHO partograph in the management of labour. Report of a WHO multicentre study 1990-1991. 1994. WHO/FHE/MSM/94.4.

4. American College of Obstetrics and Gynecology Committee on Practice Bulletins-Obstetrics. ACOG Practice Bulletin Number 49, December 2003; dystocia and augmentation of labour. Obstet Gynecol 2003;102(6):1445-1454. DOI: 10.1016/j.obstetgynecol.2003.10.011 [PubMed:14662243].

5. Zhang J, Landy HJ, Branch DW, et al. Contemporary patterns of spontaneous labour with normal neonatal outcomes. Obstet Gynecol 2010;116(6):1281-1287. DOI: 10.1097/AOG.0b013e3181fdef6e.

6. Friedman EA, Kroll BH. Computer analysis of labour progression. III. Pattern variations by parity. J Reprod Med 1971;6(4):179-183. PubMed: 4938465.

7. Friedman EA, editor. Labor: clinical evaluation and management. 2nd ed. New York: Appleton century-Crofts; 1978.

8. Peisner DB, Rosen MG. Transition from latent to active labor. Obstet Gynecol 1986;68(4);448-451. Pubmed: 3748488.

9. Albers LL, Schiff M, Gorwoda JG. The length of active labor in normal pregnancies. Obstet Gynecol 1996;87(3);355-359. DOI: 10.1016/00297844(95)00423-8. Pubmed: 8598954.

10. Albers LL. The duration of labor in healthy women. J Perinatol 1999;19(2):114-119. DOI: 10.1038/sj.jp.7200100. PubMed:10642971.

11. Jones $M$, Larson E. Length of normal labor in women of Hispanic origin. J Midwifery Womens Health 2003;48(1);2-9. DOI: 10.1016/ s1526-9523(02)00367-7. Pubmed:12589300.

12. Oladapo OT, Souza JP, Fawole B, et al. Progression of the first stage of spontaneous labour: a prospective cohort study in two sub-Saharan African countries. PLoS Med 2018;15(1): e1002492. DOI: 10.1371/ journal.pmed.1002492.

13. Suzuki R, Horiuchi S, Ohtsu H. Evaluation of the labor curve in nulliparous Japanese women. Am J Obstet Gynecol 2010;203(3):226. e1-6. DOI: 10.1016/j.ajog.2010.04.014.

14. Pitchaimuthu $\mathrm{N}$, Bhaskaran S. Labor patter among primigravida in local population. J Obstet Gynaecol India 2018;68(6);482-486. DOI: 10.1007/s13224-017-1083-3. 